\title{
Liquid biopsy of SCLC chemosensitivity
}

Small-cell lung cancer (SCLC) is arguably the most-aggressive form of lung cancer. Most patients with SCLC present with metastatic disease and have limited treatment options; although novel therapies are now on the horizon, platinum-based chemotherapy remains the current mainstay therapy. Frustratingly, most SCLCs are initially chemosensitive, but later relapse; patients with chemorefractory disease have even more dismal outcomes, and are key candidates for experimental therapies. In a new study, a novel tool has been developed that can inform on chemosensitivity prior to treatment.

Information on the molecular landscape of SCLC is limited owing to challenges in obtaining high-qualify tumour samples, which has in turn been a barrier to the identification of prognostic and predictive biomarkers, and thus to therapeutic advances. As Caroline Dive, who co-led the new study, explains, "no molecular-based tests are available to facilitate patient management, and the chemosensitive versus chemorefractory designation is based on a rather arbitrary clinical observation of a 90-day timeframe for disease relapse after chemotherapy." This situation led Dive and coworkers to probe circulating tumour cells (CTCs) from the blood for insights into the genomic landscape of SCLC. "We had previously shown that SCLC CTCs are prevalent, and developed a methodology to isolate them and profile their DNA. This provided the potential to perform a simple blood test to enable molecular analyses of tumour cells in order to increase our understanding of the disease."

Using only $7.5 \mathrm{ml}$ of blood taken from patients with SCLC prior to chemotherapy, the investigators isolated CTCs and performed next-generation sequencing of DNA from individual cells to generate genome-wide copy-number profiles. In an initial 'training set' of 88 CTCs from 13 patients, bioinformatics analysis of the genome-wide copy-number alteration (CNA) data enabled the development of a classifier, encompassing 2,281 loci that clustered within 16 CNA profiles, that predicted chemosensitivity. This classifier was tested in a validation set of 112 CTC samples from a further 18 patients. The CNA classifier correctly predicted the clinical outcome in $83 \%$ of these patients. Importantly, the patients identified as chemosensitive versus those deemed chemorefractory using the CNA classifier at baseline had substantially different progression-free and overall survival, independently of known prognostic factors, and comparable outcomes were observed for the patients deemed chemosensitive or chemorefractory based on the classic clinical criteria.

"Our data also suggest that the chemotherapy resistance that emerges after an initially good response probably occurs via different mechanisms than those observed in chemorefractory patients," Dive adds. Indeed, the researchers analysed CTCs isolated from five initially chemosensitive patients after disease progression, and demonstrated that the CNA profiles had not converted to a chemorefractory pattern at relapse.

Dive emphasizes the clinical significance of these findings: "we have developed a novel pretreatment blood test to anticipate how well and for how long a patient with SCLC will respond to chemotherapy. Knowing the depth and duration of a patient's response to first-line chemotherapy might facilitate selection of patients for clinical trials of new therapies and inform clinical trial designs. We see this as a first step towards a 'liquid biopsy'-driven approach to the management of patients with SCLC." She concludes, "a much larger study is now planned to qualify the CTC CNA profile; the profile now needs to be more thoroughly interrogated to gain clues about inherent and acquired chemotherapy resistance mechanisms."

David Killock

ORIGINAL ARTICLE Carter, L. et al. Molecular analysis of circulating tumor cells identifies distinct copy-number profiles in patients with chemosensitive and chemorefractory small-cell lung cancer. Nat.Med. http://dx.doi.org/10.1038/nm.4239 (2016) 